\title{
Reduction and duality of the generalized Hurwitz-Lerch zetas
}

\author{
Abdelmejid Bayad* and Jamel Chikhi
}

\section{"Correspondence:}

abayad@maths.univ-evry.fr

Département de mathématiques,

Université d'Evry Val d'Essonne, 23

Bd. De France, Evry Cedex, 91037,

France

\begin{abstract}
In this paper, by means of integral representation, we introduce the generalized Hurwitz-Lerch zeta functions of arbitrary complex order. For these functions, we establish the reduction formula and its associated dual formula. We then investigate analytic continuations to the whole complex plane and special values. By means of these reduction and dual formulas, we obtain nice and useful formulas for the Bernoulli-Nörlund and Apostol-Euler-Nörlund polynomials.
\end{abstract}

\section{Introduction}

The origin of the Hurwitz-Lerch zeta functions and their study go back to Riemann and Hurwitz. In fact, these zeta functions have many important identities which are at the origin of numerous applications in various areas in mathematics and physics. In this paper we introduce and investigate reduction and duality formulas for the generalized HurwitzLerch zeta functions $\zeta(s, \alpha ; x, \lambda)$. As an application, we show how these formulas can be easily used for the study of the convolution relations and computation of special values of the Apostol-Bernoulli and Apostol-Euler-Nörlund polynomials of an arbitrary order.

Throughout this paper, we use the following notations, definitions and identities.

\subsection{Notations and preliminaries}

For this subsection, we refer to Carlitz [1, 2] and Comtet [3, p.6, p.17, p.207, p.213]. Let $\alpha$ be a complex number, and let $k$ be a non-negative integer. The rising factorial $\langle\alpha\rangle_{k}$ is defined by

$$
\langle\alpha\rangle_{k}:= \begin{cases}1 & \text { if } k=0 \\ \alpha(\alpha+1) \cdots(\alpha+k-1) & \text { if } k \geq 1\end{cases}
$$

We use the falling factorial $(\alpha)_{k}=(-1)^{k}\langle-\alpha\rangle_{k}$, the binomial notation $\left\langle\begin{array}{l}\alpha \\ k\end{array}\right\rangle=\frac{\langle\alpha\rangle_{k}}{k !}$ and the polynomials $R_{1}(n, k, a)$ and $R(n, k, a)$, which are defined by equalities (1) and (2). For a nonnegative integer $n$, parameter $a$ and an indeterminate $X$, we have

$$
\langle a+X\rangle_{n}=\sum_{k=0}^{n} R_{1}(n, k, a) X^{k}
$$

๑ 2013 Bayad and Chikhi; licensee Springer. This is an Open Access article distributed under the terms of the Creative Commons Attribution License (http://creativecommons.org/licenses/by/2.0), which permits unrestricted use, distribution, and reproduction in any medium, provided the original work is properly cited. 
and

$$
X^{n}=\sum_{k=0}^{n}(-1)^{n-k} R(n, k, a)\langle a+X\rangle_{k}
$$

From Carlitz $[1,2]$ we know that these polynomials have the explicit expressions

$$
\begin{aligned}
& R_{1}(n, k, a)=\sum_{j=0}^{n-k}\left(\begin{array}{c}
j+k \\
j
\end{array}\right)|s|(n, j+k) a^{j}, \\
& R(n, k, a)=\sum_{m=0}^{n-k}\left(\begin{array}{l}
n \\
m
\end{array}\right) S(n-m, k) a^{m},
\end{aligned}
$$

where $|s|(n, l)=(-1)^{n-l} s(n, l)$, and $s(n, l)$ and $S(n, l)$ are the Stirling numbers of the first and the second kind, respectively. Moreover, these polynomials satisfy the orthogonality formulas

$$
\sum_{k=0}^{n}(-1)^{n-k} R(n, k, a) R_{1}(k, j, a)=\sum_{k=0}^{n} R_{1}(n, k, a)(-1)^{k-j} R(k, j, a)=\delta_{n, j}, \quad 0 \leq j \leq n,
$$

where $\delta_{n, j}$ is the Kronecker delta symbol.

\subsection{Motivation}

For a given positive integer $N$ and complex $\lambda, x \in \mathbb{C} \backslash\{0\}$ with $|\lambda| \leq 1$ and $\Re(x)>0$, the multiple Hurwitz-Lerch zeta function $\zeta_{N}(s ; x, \lambda)$ of order $N$ is defined by the series

$$
\zeta_{N}(s ; x, \lambda)=\sum_{k_{1}, \ldots, k_{N} \geq 0} \frac{\lambda^{k_{1}+\cdots+k_{N}}}{\left(x+k_{1}+\cdots+k_{N}\right)^{s}}
$$

for a complex $s$ such that $\Re(s)>N$ if $\lambda=1$ and $\Re(s)>0$ if $\lambda \neq 1$, and its integral representation is given by

$$
\zeta_{N}(s ; x, \lambda)=\frac{1}{\Gamma(s)} \int_{0}^{+\infty} t^{s-1} \frac{e^{-x t}}{\left(1-\lambda e^{-t}\right)^{N}} d t
$$

The ordinary Hurwitz-Lerch zeta function $\zeta(s ; x, \lambda)$, which corresponds to the function $\zeta_{1}(s ; x, \lambda)$, was originally defined in [4] by Erdelyi et al. Moreover, Choi and Srivastava [57], Kanemitsu et al. [8] and Nakamura [9] presented its various properties and applications.

The multiple Hurwitz zeta function $\zeta_{N}(s ; x)$ of order $N$ corresponds to the multiple Hurwitz-Lerch zeta function $\zeta_{N}(s ; x, 1)$, while the Hurwitz zeta function $\zeta(s ; x)$ is simply $\zeta_{1}(s ; x, 1)$. It is shown in [10] that the multiple Hurwitz zeta function $\zeta_{N}(s ; x)$ can be reduced to a finite sum of the Hurwitz zeta functions $\zeta(s ; x)$ with Stirling numbers in coefficients. Precisely, we have

$$
\zeta_{N}(s ; x)=\sum_{k=0}^{N-1} p_{N, k}(x) \zeta(s-k ; x)
$$


where

$$
p_{N, k}(x)=\frac{R_{1}(N-1, k, 1-x)}{(N-1) !} .
$$

For more details about the formula (8), see [10]. From the analytic continuations of $\zeta_{N}(s ; x)$ and $\zeta(s ; x)$ to the whole complex plane, the generalized $n$ th-Bernoulli polynomial $B_{n}^{(N)}(x)$ of order $N$ and the $n$ th-Bernoulli polynomial $B_{n}(x)$ are related to $\zeta_{N}(-n ; x)$ and $\zeta(-n ; x)$ by the formulas

$$
\begin{aligned}
& \zeta_{N}(-n ; x)=(-1)^{N} \frac{n !}{(N+n) !} B_{N+n}^{(N)}(x), \\
& \zeta(-n ; x)=-\frac{B_{n+1}(x)}{n+1},
\end{aligned}
$$

for any non-negative integer $n$. Therefore, by means of equations (8), (10) and (11), we can easily write $B_{n}^{(N)}(x)$ as a linear combination of the Bernoulli polynomials $B_{n-k}(x), k=$ $0, \ldots, N-1$ with $n \geq N$.

\subsection{Summary}

In this paper we deal with the following. Replacing the integer $N$ by any complex number $\alpha$, we relax the definition of the multiple Hurwitz-Lerch zeta function, and we generalize the formulas (8), (10) and (11). We prove reduction and duality formulas for the Hurwitz-Lerch zeta functions $\zeta(s, \alpha ; x, \lambda)$ and give applications to the Bernoulli-Nörlund and Apostol-Euler-Nörlund polynomials.

The paper can be summarized as follows. In Section 2, we state our main results. The Section 3 contains the proofs of these results. In Section 4, by means of the main results, we get reduction and its dual formulas for the Bernoulli-Nörlund and Apostol-Euler-Nörlund polynomials.

\section{Statement of main results}

Let us consider complex numbers $\alpha, \lambda$ and $x$ such that $|\lambda| \leq 1$ and $\Re(x), \Re(\alpha)>0$. We define the generalized Hurwitz-Lerch zeta function by the integral representation

$$
\begin{aligned}
& \zeta(s, \alpha ; x, \lambda) \\
& \quad=\frac{\Gamma(\alpha)}{\Gamma(s)} \int_{0}^{\infty} \frac{t^{s-1} e^{-\alpha t}}{\left(1-\lambda e^{-t}\right)^{\alpha}} d t, \quad \Re(s)>\Re(\alpha) \text { for } \lambda=1 \text { and } \Re(s)>0 \text { for } \lambda \neq 1 .
\end{aligned}
$$

Lemma 2.1 Let $\tau$ be a positive real number, and let $\alpha, \lambda$ be complex numbers such that $|\lambda| \leq 1$. Then the series of functions

$$
\sum_{k=0}^{\infty}\left(\begin{array}{l}
\alpha \\
k
\end{array}\right) \lambda^{k} e^{-k t}
$$

is absolutely and uniformly convergent on $[\tau,+\infty[$ and

$$
\frac{1}{\left(1-\lambda e^{-t}\right)^{\alpha}}=\sum_{k=0}^{\infty}\left(\begin{array}{l}
\alpha \\
k
\end{array}\right) \lambda^{k} e^{-k t} .
$$


Proof Indeed, we have for all positive integer $k$ the majoration

$$
\left|\left(\begin{array}{l}
\alpha \\
k
\end{array}\right) \lambda^{k} e^{-k t}\right| \leq \frac{\langle|\alpha|\rangle_{k}}{k !} e^{-k \tau}
$$

and the ratio

$$
\frac{\langle|\alpha|\rangle_{k+1} e^{-(k+1) \tau}}{(k+1) !} \cdot \frac{k !}{\langle|\alpha|\rangle_{k} e^{-k \tau}}
$$

tends to $\left.e^{-\tau} \in\right] 0,1[$ as $k \rightarrow+\infty$. Thus, the lemma is proved.

On the other hand, the integral

$$
\int_{0}^{\infty} \frac{t^{s-1} e^{-x t}}{\left(1-\lambda e^{-t}\right)^{\alpha}} d t=\int_{0}^{\infty} t^{s-1} e^{-x t}\left(\sum_{k=0}^{\infty}\left(\begin{array}{l}
\alpha \\
k
\end{array}\right) \lambda^{k} e^{-k t}\right) d t
$$

is absolutely convergent for $\Re(s)>\Re(\alpha)$ for $\lambda=1$, and $\Re(s)>0$ for $\lambda \neq 1$. Therefore, by means of Lemma 2.1, we can interchange the summation and integration to obtain

$$
\int_{0}^{\infty} \frac{t^{s-1} e^{-x t}}{\left(1-\lambda e^{-t}\right)^{\alpha}} d t=\sum_{k=0}^{\infty}\left(\begin{array}{l}
\alpha \\
k
\end{array}\right) \lambda^{k}\left(\int_{0}^{\infty} t^{s-1} e^{-(x+k) t} d t\right)=\Gamma(s) \sum_{k=0}^{\infty}\left(\begin{array}{l}
\alpha \\
k
\end{array}\right) \frac{\lambda^{k}}{(x+k)^{s}}
$$

Therefore, we obtain the series representation of $\zeta(s, \alpha ; x, \lambda)$ as follows.

Proposition 2.2 For any complex numbers $\alpha, \lambda$ and $x$ such that $|\lambda| \leq 1$ and $\Re(x), \Re(\alpha)>0$, we have

$$
\zeta(s, \alpha ; x, \lambda)=\sum_{k=0}^{\infty} \frac{\Gamma(\alpha+k)}{k !} \cdot \frac{\lambda^{k}}{(x+k)^{s}} .
$$

Note that for $\alpha$ be a positive integer $N$, we have $\zeta_{N}(s ; x, \lambda)=\frac{\zeta(s, N ; x, \lambda)}{(N-1) !}$, and by Proposition 2.2, their series representations are given as follows.

Corollary 2.3 For any positive integer $N$, complex number $\lambda$ and $x$ such that $|\lambda| \leq 1$ and $\Re(x)>0$, we have

$$
\begin{aligned}
& \zeta_{N}(s ; x, \lambda) \\
& \quad=\sum_{k=0}^{\infty}\left(\begin{array}{c}
k+N-1 \\
N-1
\end{array}\right) \frac{\lambda^{k}}{(x+k)^{s}}, \quad \text { where } \Re(s)>N \text { for } \lambda=1 \text { and } \Re(s)>0 \text { for } \lambda \neq 1 .
\end{aligned}
$$

We are now able to state our main results.

Theorem 2.4 (Reduction formula) For any non-negative integer $N$ and complex numbers $\alpha, \lambda$, x such that $|\lambda| \leq 1$ and $\Re(x), \Re(\alpha)>0$, the following reduction formula holds:

$$
\zeta(s, \alpha+N ; x, \lambda)=\sum_{k=0}^{N} R_{1}(N, k, \alpha-x) \zeta(s-k, \alpha ; x, \lambda),
$$

with $\Re(s)>\Re(\alpha)+N$ for $\lambda=1$ and $\Re(s)>N$ for $\lambda \neq 1$. 
By dualizing the above theorem, we obtain the following formula.

Theorem 2.5 (Duality formula) For any non-negative integer $N$ and complex numbers $\alpha$, $\lambda$, $x$ such that $|\lambda| \leq 1$ and $\Re(x), \Re(\alpha)>0$, we have

$$
\zeta(s-N, \alpha ; x, \lambda)=\sum_{k=0}^{N}(-1)^{N-k} R(N, k, \alpha-x) \zeta(s, \alpha+k ; x, \lambda)
$$

with $\mathfrak{R}(s)>\mathfrak{R}(\alpha)+N$ for $\lambda=1$ and $\Re(s)>N$ for $\lambda \neq 1$.

For $\alpha=1$, we get an extension of Choi's reduction formula to multiple Hurwitz-Lerch zeta $\zeta_{N}(s ; x, \lambda)$, and we find its dual version.

Corollary 2.6 Let $N$ be a positive integer, and let $x, \lambda$ be complex numbers such that $|\lambda| \leq 1$, $\Re(x)>0$. Then, for any complex $s$ with $\Re(s)>N$, we have the reduction and duality formulas

$$
\begin{aligned}
& \zeta_{N}(s ; x, \lambda)=\sum_{k=0}^{N-1} \frac{R_{1}(N-1, k, 1-x)}{(N-1) !} \zeta(s-k ; x, \lambda), \\
& \zeta(s-N ; x, \lambda)=\sum_{k=0}^{N}(-1)^{N-k} k ! R(N, k, 1-x) \zeta_{k+1}(s ; x, \lambda) .
\end{aligned}
$$

Substituting $x=\lambda=1$ in Corollary 2.6, we obtain the following.

Corollary 2.7 Let $N$ be a positive integer. For any complex $s$ with $\Re(s)>N$, we obtain the formula

$$
\zeta_{N}(s)=\frac{1}{(N-1) !} \sum_{k=0}^{N-1}|s|(N-1, k) \zeta(s-k)
$$

and its dual

$$
\zeta(s-N)=\sum_{k=0}^{N} k !(-1)^{N-k} S(N, k) \zeta_{k+1}(s)
$$

where $\zeta_{N}(s)=\frac{\zeta(s, N ; 1)}{(N-1) !}$ is the Riemann zeta function of order $N$.

\section{Proofs of Theorem 2.4 and Theorem 2.5}

In the sequel, all the parameters under consideration are subject to the conditions of the theorem. For fixed parameters $\alpha, x$ and $\lambda$, the function $t>0 \mapsto f(t, \alpha ; x, \lambda):=\Gamma(\alpha) e^{-x t}(1-$ $\left.\lambda e^{-t}\right)^{-\alpha}$ is smooth and satisfies the differential identity

$$
\partial_{t} f(t, \alpha ; x, \lambda)=(\alpha-x) f(t, \alpha ; x, \lambda)-f(t, \alpha+1 ; x, \lambda) .
$$

From the identity (18), with the help of integration by parts, we get

$$
\begin{aligned}
& \Gamma(s) \zeta(s, \alpha+1 ; x, \lambda) \\
& \quad=\int_{0}^{\infty} t^{s-1} f(t, \alpha+1 ; x, \lambda) d t
\end{aligned}
$$




$$
\begin{aligned}
& =(\alpha-x) \int_{0}^{\infty} t^{s-1} f(t, \alpha ; x, \lambda) d t-\int_{0}^{\infty} t^{s-1} \partial_{t} f(t, \alpha ; x, \lambda) d t \\
& =(\alpha-x) \int_{0}^{\infty} t^{s-1} f(t, \alpha ; x, \lambda) d t-\left[t^{s-1} f_{\alpha}(t)\right]_{0}^{\infty}+(s-1) \int_{0}^{\infty} t^{s-2} f(t, \alpha ; x, \lambda) d t .
\end{aligned}
$$

The hypotheses of the theorems ensure that $\left[t^{s-1} f(t, \alpha ; x, \lambda)\right]_{0}^{\infty}=0$, and hence we have

$$
\begin{aligned}
\Gamma(s) \zeta(s, \alpha+1 ; x, \lambda) & =(\alpha-x) \int_{0}^{\infty} t^{s-1} f(t, \alpha ; x, \lambda) d t+(s-1) \int_{0}^{\infty} t^{s-2} f(t, \alpha ; x, \lambda) d t \\
& =(\alpha-x) \Gamma(s) \zeta(s, \alpha ; x, \lambda)+(s-1) \Gamma(s-1) \zeta(s-1, \alpha ; x, \lambda),
\end{aligned}
$$

and therefore

$$
\zeta(s, \alpha+1 ; x, \lambda)=(\alpha-x) \zeta(s, \alpha ; x, \lambda)+\zeta(s-1, \alpha ; x, \lambda)
$$

or, equivalently,

$$
\left(\alpha-x+E_{s}^{-}\right) \zeta(s, \alpha ; x, \lambda)=E_{\alpha}^{+} \zeta(s, \alpha ; x, \lambda)
$$

where the functions $E_{u}^{ \pm}: \varphi(u) \mapsto \varphi(u \pm 1)$ are the translation operators. By switching $\alpha$ in the identity (19), we see that the $N$ equalities

$$
\left(\alpha+k-x+E_{s}^{-}\right) \zeta(s, \alpha+k ; x, \lambda)=E_{\alpha}^{+} \zeta(s, \alpha+k ; x, \lambda), \quad k=0,1, \ldots, N-1
$$

hold. The composition of these equalities yields the relation

$$
\left\langle\alpha-x+E_{s}^{-}\right\rangle_{N} \zeta(s, \alpha ; x, \lambda)=\left(E_{\alpha}^{+}\right)^{N} \zeta(s, \alpha ; x, \lambda)
$$

On the other hand, we have

$$
\begin{aligned}
& \left\langle\alpha-x+E_{s}^{-}\right\rangle_{N}=\sum_{k=0}^{N} R_{1}(N, k, \alpha-x)\left(E_{s}^{-}\right)^{k}, \\
& \left(E_{\alpha}^{+}\right)^{N} \zeta(s, \alpha ; x, \lambda)=\zeta(s, \alpha+N ; x, \lambda) .
\end{aligned}
$$

Therefore, from equalities (19), (23) and (24), we get our Theorem 2.4.

The proof of Theorem 2.5 is an immediate consequence of the orthogonality properties (5) of the polynomials $R(n, k, a)$ and $R_{1}(k, j, a)$, and Theorem 2.4.

\section{The Bernoulli-Nörlund and Apostol-Euler-Nörlund polynomials}

\subsection{Apostol-Euler-Nörlund polynomials}

The Apostol-Euler-Nörlund polynomials $E_{n}^{(\alpha)}(x ; \lambda)$ are defined, for $\lambda \neq-1$, by the generating function

$$
\left(\frac{2}{\lambda e^{t}+1}\right)^{\alpha} e^{x t}=\sum_{n=0}^{\infty} E_{n}^{(\alpha)}(x ; \lambda) \frac{t^{n}}{n !}, \quad|t|<|\log (-\lambda)| .
$$


We consider complex numbers $\alpha, \lambda$ and $x$ such that $|\lambda| \leq 1$ and $\Re(x), \Re(\alpha)>0$. We first prove the analytic continuation of $s \mapsto \Gamma(s) \zeta(s, \alpha ; x, \lambda)$, and we compute special values of $\zeta(s, \alpha ; x, \lambda)$.

Theorem 4.1 Let $\lambda$ be a complex number with $\lambda \neq 1$. The function $s \mapsto \Gamma(s) \zeta(s, \alpha ; x, \lambda)$ has analytic continuation to the whole complex plane, except possible simple poles at nonpositive integers $s=-m$ with residue

$$
\frac{\Gamma(\alpha)}{m !} E_{m}^{(\alpha)}(x ;-\lambda)
$$

Moreover, the function $s \mapsto \zeta(s, \alpha ; x, \lambda)$ has analytic continuation as an entire function to the whole complex plane, and for all non-negative integer $n$, we have

$$
\Gamma(\alpha) E_{n}^{(\alpha)}(x ;-\lambda)=2^{\alpha} \zeta(-n, \alpha ; x, \lambda)
$$

Proof Let $\lambda$ be a complex number other than 1 . We choose a real number $\delta>0$ so that $\delta<|\log \lambda|$. We split the integral from zeta's definition as

$$
\frac{\Gamma(s)}{\Gamma(\alpha)} \zeta(s, \alpha ; x, \lambda)=\left(\int_{0}^{\delta}+\int_{\delta}^{\infty}\right) t^{s-1} \frac{e^{-x t}}{\left(1-\lambda e^{-t}\right)^{\alpha}} d t
$$

As $\Re(x)>0$ and $|\lambda| \leq 1$, for all complex number $\alpha$, the integral

$$
\int_{\delta}^{\infty} t^{s-1} \frac{e^{-x t}}{\left(1-\lambda e^{-t}\right)^{\alpha}} d t
$$

defines an entire function of $s \in \mathbb{C}$.

We substitute in the first integral, for $\Re(s)>0$, the generating expansion of the ApostolEuler-Nörlund polynomials

$$
2^{\alpha} \int_{0}^{\delta} t^{s-1} \frac{e^{-x t}}{\left(1-\lambda e^{-t}\right)^{\alpha}} d t=\sum_{n=0}^{\infty} \frac{(-1)^{n} E_{n}^{(\alpha)}(x ;-\lambda)}{n !} \int_{0}^{\delta} t^{s-1+n} d t=\sum_{n=0}^{\infty} \frac{(-1)^{n} \delta^{s+n}}{n !(s+n)} E_{n}^{(\alpha)}(x ;-\lambda) .
$$

It hence follows that

$$
\frac{2^{\alpha}}{\Gamma(\alpha)} \zeta(s, \alpha ; x, \lambda)=\sum_{n=0}^{\infty} \frac{(-1)^{n} \delta^{s+n}}{n !(s+n) \Gamma(s)} E_{n}^{(\alpha)}(x ;-\lambda)+\frac{2^{\alpha}}{\Gamma(s)} \int_{\delta}^{\infty} t^{s-1} \frac{e^{-x t}}{\left(1-\lambda e^{-t}\right)^{\alpha}} d t
$$

For a given non-negative integer $m$, we know that

$$
\lim _{s \rightarrow-m}(s+m) \Gamma(s)=\frac{(-1)^{m}}{m !}
$$

and $1 / \Gamma(-m)=0$. This proves the analytic continuation of the function $s \mapsto \zeta(s, \alpha ; x, \lambda)$ as an entire function to the whole complex plane, and

$$
\Gamma(\alpha) E_{m}^{(\alpha)}(x ;-\lambda)=2^{\alpha} \zeta(-m, \alpha ; x, \lambda) .
$$


Remark 4.2 Equality (28) has been proved, using different method, by Luo [11, Theorem 2.1].

Now, by applying our Theorem 2.4, Theorem 2.5 and Theorem 4.1, we deduce the reduction and duality formulas for the Apostol-Euler-Nörlund polynomials $E_{n}^{(\alpha)}(x ; \lambda)$.

Theorem 4.3 For any non-negative integers $n, N$ and complex numbers $\alpha, \lambda \neq-1$ with $|\lambda| \leq 1$, we have

$$
2^{-N}\langle\alpha\rangle_{N} E_{n}^{(\alpha+N)}(x ; \lambda)=\sum_{k=0}^{N} R_{1}(N, k, \alpha-x) E_{n+k}^{(\alpha)}(x ; \lambda)
$$

and

$$
E_{n+N}^{(\alpha)}(x ; \lambda)=\sum_{k=0}^{N}(-1)^{N-k} R(N, k, \alpha-x) 2^{-k}\langle\alpha\rangle_{k} E_{n}^{(\alpha+k)}(x ; \lambda) .
$$

\subsection{Explicit formula for the Apostol-Euler-Nörlund polynomials}

In particular for $n=0$, and by using formula (29), we get this explicit formula for the Apostol-Euler-Nörlund polynomials.

Proposition 4.4 Let $\alpha, \lambda$ be complex numbers with $|\lambda| \leq 1, \lambda \neq-1$. For any positive integer $N$, we have

$$
E_{N}^{(\alpha)}(x ; \lambda)=\frac{2^{\alpha}}{(\lambda+1)^{\alpha}} \sum_{k=0}^{N}(-1)^{N-k} R(N, k, \alpha-x) \frac{\langle\alpha\rangle_{k}}{(\lambda+1)^{k}} .
$$

The above formula, when combined with the well-known equality

$$
E_{N}^{(\alpha)}(x ; \lambda)=\sum_{k=0}^{N}\left(\begin{array}{c}
N \\
k
\end{array}\right) x^{N-k} E_{k}^{(\alpha)}(0 ; \lambda),
$$

gives this other explicit expression

$$
E_{N}^{(\alpha)}(x ; \lambda)=\frac{2^{\alpha}}{(\lambda+1)^{\alpha}} \sum_{k=0}^{N}\left(\begin{array}{c}
N \\
k
\end{array}\right) x^{N-k}\left(\sum_{j=0}^{k}(-1)^{k-j} R(k, j, \alpha) \frac{\langle\alpha\rangle_{j}}{(\lambda+1)^{j}}\right) .
$$

\subsection{Differential formula for the Apostol-Euler-Nörlund polynomials}

We consider the differential operator $D_{\lambda}=\lambda \frac{d}{d \lambda}$. From the series representation (2.2) of the Hurwitz-Lerch zeta functions, we have

$$
D_{\lambda}^{n}\left(\lambda^{x} \zeta(s, \alpha ; x, \lambda)\right)=\lambda^{x} \zeta(s-n, \alpha ; x, \lambda)
$$

Using Theorem 4.1 at $s=0$, we obtain the differential formula

$$
E_{n}^{(\alpha)}(x ; \lambda)=2^{\alpha} \lambda^{-x} D_{\lambda}^{n}\left(\frac{\lambda^{x}}{(1+\lambda)^{\alpha}}\right) .
$$




\subsection{Bernoulli-Nörlund polynomials}

In this subsection we investigate convolution formulas for the Bernoulli-Nörlund polynomials $B_{n}^{(\alpha)}(x)$. We define them by the generating function

$$
\left(\frac{t}{e^{t}-1}\right)^{\alpha} e^{x t}=\sum_{n=0}^{\infty} B_{n}^{(\alpha)}(x) \frac{t^{n}}{n !}, \quad|t|<2 \pi .
$$

The Nörlund polynomials are $B_{n}^{(\alpha)}:=B_{n}^{(\alpha)}(0)$, see [12].

We set $a=\Re(\alpha)$, and we introduce the modified Hurwitz-Lerch zeta function defined by the integral representation

$$
\zeta^{\prime \prime}(s, \alpha ; x)=\frac{1}{\Gamma(s-\alpha+[a])} \int_{0}^{\infty} \frac{t^{s-1} e^{-x t}}{\left(1-e^{-t}\right)^{\alpha}} d t, \quad \Re(s)>\max (a,\{a\}) .
$$

Theorem $4.5(\lambda=1)$ Let $\alpha, x$ be complex numbers with $\Re(x)>0$. The function $s \mapsto \Gamma(s-$ $\alpha+[a]) \zeta "(s, \alpha ; x)$ has analytic continuation to the whole complex plane, except simple poles at $s=\alpha-m$ with residue $\frac{(-1)^{m}}{m !([a]-m-1) !} B_{m}^{(\alpha)}(x)$, for any non-negative integer $m<[a]$ (when $a \geq 1$ ). Moreover, for all non-negative integer $m \geq[a]$, we have

$$
\zeta^{\prime \prime}(\alpha-m, \alpha ; x)=(-1)^{[a]} \frac{(m-[a]) !}{m !} B_{m}^{(\alpha)}(x) .
$$

Proof The proof is similar to that of Theorem 4.1. We split the integral

$$
\Gamma(s-\alpha+[a]) \zeta^{*}(s, \alpha ; x)=\left(\int_{0}^{1}+\int_{1}^{\infty}\right) t^{s-1} \frac{e^{-x t}}{\left(1-e^{-t}\right)^{\alpha}} d t .
$$

As $\Re(x)>0$, for all complex number $\alpha$, the integral

$$
\int_{1}^{\infty} t^{s+\alpha-1} \frac{e^{-x t}}{\left(1-e^{-t}\right)^{\alpha}} d t
$$

defines an entire function of $s \in \mathbb{C}$.

We use in the first integral, for $\Re(s)>a$, the generating function of the BernoulliNörlund polynomials

$$
\int_{0}^{1} t^{s-1} \frac{e^{-x t}}{\left(1-e^{-t}\right)^{\alpha}} d t=\sum_{n=0}^{\infty} \frac{(-1)^{n}}{n !} B_{n}^{(\alpha)}(x) \int_{0}^{1} t^{s-\alpha-1+n} d t=\sum_{n=0}^{\infty} \frac{(-1)^{n}}{n !(s-\alpha+n)} B_{n}^{(\alpha)}(x) .
$$

It hence follows that

$$
\zeta^{\prime \prime}(s, \alpha ; x)=\sum_{n=0}^{\infty} \frac{(-1)^{n}}{n !(s-\alpha+n) \Gamma(s-\alpha+[a])} B_{n}^{(\alpha)}(x)+\frac{1}{\Gamma(s-\alpha+[a])} \int_{1}^{\infty} t^{s-1} \frac{e^{-x t}}{\left(1-e^{-t}\right)^{\alpha}} d t .
$$

For a given non-negative integer $n<[a]$ (if $[a]>0$ ), we have a simple pole at $s=\alpha-n$ with residue $\frac{(-1)^{n}}{n !([a]-n-1) !} B_{n}^{(\alpha)}(x)$.

For a given integer $n \geq[a]$, it is known that

$$
\lim _{s \rightarrow \alpha-n}(s-\alpha+n) \Gamma(s-\alpha+[a])=\frac{(-1)^{[a]-n}}{(n-[a]) !}
$$


and $1 / \Gamma([a]-n)=0$. Then we obtain

$$
B_{m}^{(\alpha)}(x)=\langle-m\rangle_{[a]} \zeta^{*}(\alpha-m, \alpha ; x)
$$

This proves the analytic continuation of the function $s \mapsto \zeta^{*}(s, \alpha ; x)$ as an entire function to the whole complex plane, except simple poles at $s=\alpha-n, 0 \leq n<[a]$ if $\Re(\alpha) \geq 1$.

This completes the proof of the theorem.

Remark 4.6 For any positive integer $\alpha$, the relation (35) recovers the results in the paper [13].

Observe that

$$
\Gamma(\alpha) \zeta^{*}(s, \alpha ; x)=\frac{\Gamma(s)}{\Gamma(s-\alpha+[a])} \zeta(s, \alpha ; x, 1)
$$

Hence, from Theorem 2.4 and Theorem 4.1, we deduce the following reduction and duality formulas.

Theorem 4.7 Under the hypothesis of Theorem 2.4, we have

$$
\begin{aligned}
& \langle\alpha\rangle_{N} \zeta^{*}(s, \alpha+N ; x)=\sum_{k=0}^{N} R_{1}(N, k, \alpha-x) \frac{(s-1)_{k}}{(s-1-\alpha+[a])_{k}} \zeta^{*}(s-k, \alpha ; x), \\
& \frac{(s-1)_{N}}{(s-1-\alpha+[a])_{N}} \zeta^{*}(s-N, \alpha ; x)=\sum_{k=0}^{N}(-1)^{N-k} R(N, k, \alpha-x)\langle\alpha\rangle_{k} \zeta^{*}(s, \alpha+k ; x) .
\end{aligned}
$$

By use of Theorem 4.1 and equalities (36), (37), we get the convolution identities on the Bernoulli-Nörlund polynomials.

Theorem 4.8 For any non-negative integer $n$ and any positive integer $N$, we have the convolution identity and its dual version

$$
\begin{aligned}
& \langle\alpha\rangle_{N} \frac{B_{n+N}^{(\alpha+N)}(x)}{(n+N) !}=(-1)^{N} \sum_{k=0}^{N} R_{1}(N, k, \alpha-x)\langle n+1-\alpha\rangle_{k} \frac{B_{n+k}^{(\alpha)}(x)}{(n+k) !}, \\
& \langle n+1-\alpha\rangle_{N} \frac{B_{n+N}^{(\alpha)}(x)}{(n+N) !}=\sum_{k=0}^{N} R(N, k, \alpha-x)\langle\alpha\rangle_{k} \frac{B_{n+k}^{(\alpha+k)}(x)}{(n+k) !} .
\end{aligned}
$$

Note that from Theorem 4.8 we have the following corollaries.

Corollary 4.9 Under the hypothesis of Theorem 4.8, for $x=0$, and indeterminate $\alpha$, we obtain, among the so-called Nörlund polynomials $B_{n}^{(\alpha)}$, the formula

$$
\langle\alpha\rangle_{N} \frac{B_{n+N}^{(\alpha+N)}}{(n+N) !}=(-1)^{N} \sum_{k=0}^{N} R_{1}(N, k, \alpha)\langle n+1-\alpha\rangle_{k} \frac{B_{n+k}^{(\alpha)}}{(n+k) !} .
$$


Corollary 4.10 Under the hypothesis of Theorem 4.8, for $x=\alpha$, we obtain the following formula:

$$
\langle\alpha\rangle_{N} \frac{B_{n+N}^{(\alpha+N)}(\alpha)}{(n+N) !}=\sum_{k=0}^{N} s(N, k)\langle\alpha-k-n\rangle_{k} \frac{B_{n+k}^{(\alpha)}(\alpha)}{(n+k) !} .
$$

Corollary 4.11 Under the hypothesis of Theorem 4.8, and if $x=0, \alpha=1$, we obtain Euler's identity type on the Bernoulli numbers of order $N$

$$
\frac{B_{n+N-1}^{(N)}}{(n+N-1) !}=\sum_{k=0}^{N-1} s(N, k+1)\langle 1-k-n\rangle_{k} \frac{B_{n+k}}{(n+k) !} .
$$

This formula is an analogue of the nice identity for Bernoulli numbers obtained by Euler and given by

$$
B_{n+1}^{(2)}=-(n+1) B_{n}-n B_{n+1} \quad(n \geq 0)
$$

and its generalization to Bernoulli numbers $B_{n+1}^{(N)}$ of arbitrary level $N$, cf. $[14,15]$.

Corollary 4.12 Under the hypothesis of Theorem 4.8, taking $n=0$ in the dual formula (39), we obtain

$$
\langle 1-\alpha\rangle_{N} \frac{B_{N}^{(\alpha)}(x)}{N !}=\sum_{k=0}^{N} R(N, k, \alpha-x)\langle\alpha\rangle_{k} \frac{B_{k}^{(\alpha+k)}(x)}{k !} .
$$

\section{Further applications}

We briefly indicate some possible ways to generalize a few known special functions related to the Hurwitz-Lerch zetas functions. We give, in addition, associated reduction and duality formulas.

\subsection{Generalized polylogarithms}

Let $\alpha, \lambda$ be complex numbers such that $|\lambda| \leq 1$ and $\Re(\alpha)>0$. We define the generalized polylogarithms by the equality

$$
\operatorname{Li}(s, \alpha ; \lambda)=\sum_{k=1}^{\infty} \frac{\Gamma(\alpha+k-1)}{(k-1) !} \cdot \frac{\lambda^{k}}{k^{s}}
$$

which is equivalent to the equalities

$$
\operatorname{Li}(s, \alpha ; \lambda)=\lambda \zeta(s, \alpha ; 1, \lambda)
$$

and

$$
\operatorname{Li}(s, \alpha ; \lambda)=\frac{\lambda \Gamma(\alpha)}{\Gamma(s)} \int_{0}^{\infty} \frac{t^{s-1} e^{-t}}{\left(1-\lambda e^{-t}\right)^{\alpha}} d t
$$

for $\Re(s)>\Re(\alpha)$ if $\lambda=1$ and $\Re(s)>0$ if $\lambda \neq 1$. 
The ordinary polylogarithm corresponds to

$$
\operatorname{Li}(s ; \lambda)=\operatorname{Li}(s, 1 ; \lambda)=\frac{\lambda}{\Gamma(s)} \int_{0}^{\infty} \frac{t^{s-1}}{e^{t}-\lambda} d t
$$

for more details, see $[16,17]$.

Therefore, from Theorem 2.4 and Theorem 2.5, the following reduction and duality formulas hold.

Theorem 5.1 Under the hypothesis of Theorem 2.4 on the parameters $\alpha, \lambda, N$ and $s$, we have the reduction and duality relations for the generalized polylogarithms

$$
\begin{aligned}
& \operatorname{Li}(s, \alpha+N ; \lambda)=\sum_{k=0}^{N} R_{1}(N, k, \alpha-1) \operatorname{Li}(s-k, \alpha ; \lambda), \\
& \operatorname{Li}(s-N, \alpha ; \lambda)=\sum_{k=0}^{N}(-1)^{N-k} R(N, k, \alpha-1) \operatorname{Li}(s, \alpha+k ; \lambda) .
\end{aligned}
$$

\subsection{Generalized Fermi-Dirac functions}

Following Srivastava et al. [18], we extend the definition of the Fermi-Dirac functions, with parameter $\alpha$ with $\mathfrak{R}(\alpha)>0$, as follows:

$$
\Theta_{v}(s, \alpha ; x)=\frac{\Gamma(\alpha)}{\Gamma(s)} \int_{0}^{\infty} t^{s-1} \frac{e^{-v(x+t)}}{\left(e^{x+t}+1\right)^{\alpha}} d t, \quad \Re(x) \geq 0, \Re(v)>-\Re(\alpha),
$$

and $\Re(s)>\Re(\alpha)$ if $e^{-x}=-1$ and $\Re(s)>0$ if $e^{-x} \neq-1$. Alternatively, they have a series representation related to the Hurwitz-Lerch zetas. Under the same conditions on parameters as above, we have

$$
\begin{aligned}
& \Theta_{v}(s, \alpha ; x)=\sum_{k=1}^{\infty} \frac{\Gamma(\alpha+k)}{k !} \cdot \frac{(-1)^{k} e^{-x(v+\alpha+k)}}{(v+\alpha+k)^{s}}, \\
& \Theta_{v}(s, \alpha ; x)=e^{-x(v+\alpha)} \zeta\left(s, \alpha ; v+\alpha,-e^{-x}\right) .
\end{aligned}
$$

The ordinary Fermi-Dirac function is given by

$$
\Theta_{v}(s ; x):=\Theta_{v}(s, 1 ; x)=\frac{1}{\Gamma(s)} \int_{0}^{\infty} t^{s-1} \frac{e^{-v(x+t)}}{e^{x+t}+1} d t .
$$

Theorem 5.2 We have the reduction and duality relations for the generalized Fermi-Dirac functions

$$
\begin{aligned}
& \Theta_{v-N}(s, \alpha+N ; x)=\sum_{k=0}^{N} R_{1}(N, k,-v) \Theta_{v}(s-k, \alpha ; x), \\
& \Theta_{v}(s-N, \alpha ; x)=\sum_{k=0}^{N}(-1)^{N-k} R(N, k,-v) \Theta_{v-k}(s, \alpha+k ; x),
\end{aligned}
$$

for $\mathfrak{R}(x) \geq 0, \mathfrak{R}(v)>-\mathfrak{H}(\alpha), \mathfrak{R}(s)>\mathfrak{N}(\alpha)+N$ if $e^{-x}=-1$, and $\mathfrak{R}(s)>N$ otherwise. 


\subsection{Generalized Bose-Einstein functions}

The generalized Bose-Einstein functions can also be defined as follows. As the case $\alpha=1$ in [18], we can define them by their integral representations

$$
\Psi_{v}(s, \alpha ; x)=\frac{\Gamma(\alpha)}{\Gamma(s)} \int_{0}^{\infty} t^{s-1} \frac{e^{-v(x+t)}}{\left(e^{x+t}-1\right)^{\alpha}} d t, \quad \Re(x) \geq 0, \Re(v)>-\Re(\alpha)
$$

and $\Re(s)>\Re(\alpha)$ if $e^{-x}=1$, and $\Re(s)>0$ otherwise. Alternatively, their series representation and relationship with Hurwitz-Lerch zetas are given by the equalities

$$
\begin{aligned}
& \Psi_{v}(s, \alpha ; x)=\sum_{k=1}^{\infty} \frac{\Gamma(\alpha+k)}{k !} \cdot \frac{e^{-x(v+\alpha+k)}}{(v+\alpha+k)^{s}}, \\
& \Psi_{v}(s, \alpha ; x)=e^{-x(v+\alpha)} \zeta\left(s, \alpha ; v+\alpha, e^{-x}\right) .
\end{aligned}
$$

The ordinary Bose-Einstein function corresponds to

$$
\Psi_{v}(s ; x):=\Psi_{\nu}(s, 1 ; x)=\frac{1}{\Gamma(s)} \int_{0}^{\infty} t^{s-1} \frac{e^{-v(x+t)}}{e^{x+t}-1} d t
$$

The related reduction and duality formulas are then similar to those of the generalized Fermi-Dirac functions.

Theorem 5.3 We have the reduction and duality relations for the generalized BoseEinstein functions

$$
\begin{aligned}
& \Psi_{v-N}(s, \alpha+N ; x)=\sum_{k=0}^{N} R_{1}(N, k,-v) \Psi_{v}(s-k, \alpha ; x), \\
& \Psi_{\nu}(s-N, \alpha ; x)=\sum_{k=0}^{N}(-1)^{N-k} R(N, k,-v) \Psi_{v-k}(s, \alpha+k ; x),
\end{aligned}
$$

for $\Re(x) \geq 0, \Re(v)>-\Re(\alpha), \Re(s)>\Re(\alpha)+N$ if $e^{-x}=1$, and $\Re(s)>N$ otherwise.

\subsection{Formulas for the generalized Euler-Frobenius polynomials}

We consider the Apostol-Euler-Frobenius-Nörlund type polynomials $H_{n}^{(\alpha)}(x ; \lambda \mid u)$ defined as follows.

For $u \neq 0,1$ and $\lambda \neq 0, u$, the Frobenius-Euler-Nörlund polynomials are defined through the generating function

$$
\left(\frac{1-u}{\lambda e^{t}-u}\right)^{\alpha} e^{x t}:=\sum_{n=0}^{\infty} H_{n}^{(\alpha)}(x ; \lambda \mid u) \frac{t^{n}}{n !}, \quad|t|<\left|\log \left(\frac{\lambda}{u}\right)\right| .
$$

The so-called Euler-Frobenius polynomials correspond to $H_{n}(x \mid u):=H_{n}^{(1)}(x ; 1 \mid u)$, and we denote the Apostol-Euler-Frobenius polynomials by $H_{n}(x ; \lambda \mid u):=H_{n}^{(1)}(x ; \lambda \mid u)$.

By writing

$$
\left(\frac{1-u}{\lambda e^{t}-u}\right)^{\alpha}=\left(\frac{u-1}{2 u}\right)^{\alpha}\left(\frac{2}{\frac{-\lambda}{u} e^{t}+1}\right)^{\alpha}
$$


we easily see that for all non-negative integer $n$, we have

$$
H_{n}^{(\alpha)}(x ; \lambda \mid u)=\left(\frac{u-1}{2 u}\right)^{\alpha} E_{n}^{(\alpha)}\left(x,-\frac{\lambda}{u}\right)
$$

and using the explicit formula (30) of the Apostol-Euler-Nörlund polynomials, we get that of the Apostol-Euler-Frobenius-Nörlund polynomials

$$
H_{n}^{(\alpha)}(x ; \lambda \mid u)=\left(\frac{u-1}{u-\lambda}\right)^{\alpha} \sum_{k=0}^{n}\left(\begin{array}{l}
n \\
k
\end{array}\right) x^{n-k}\left(\sum_{j=0}^{k}(-1)^{k-j} R(k, j, \alpha)\langle\alpha\rangle_{j}\left(\frac{u}{u-\lambda}\right)^{j}\right) .
$$

On the other hand, using equality (33), we deduce the differential formula for the ApostolEuler-Frobenius-Nörlund polynomials

$$
H_{n}^{(\alpha)}(x ; \lambda \mid u)=(u-1)^{\alpha} \lambda^{-x} D_{\lambda}^{n}\left(\frac{\lambda^{x}}{(u-\lambda)^{\alpha}}\right) .
$$

\section{Competing interests}

The authors declare that they have no competing interests.

\section{Authors' contributions}

All authors completed the paper, read and approved the final manuscript.

\section{Acknowledgements}

Dedicated to Professor Hari M Srivastava.

The present investigation was supported by the 'Equipe Ananlyse et Probabilités' of the Department of Mathematics at University of Evry.

Received: 14 December 2012 Accepted: 15 March 2013 Published: 4 April 2013

\section{References}

1. Carlitz, L: Weighted Stirling numbers of the first and second kind - I. Fibonacci Q. 18, 147-162 (1980)

2. Carlitz, L: Weighted Stirling numbers of the first and second kind - II. Fibonacci Q. 18, 242-257 (1980)

3. Comtet, L: Advanced Combinatorics. Reidel, Dordrecht (1974)

4. Erdelyi, A: Higher Transcendental Functions, vol. 1. McGraw Hill, New York (1953)

5. Choi, J, Srivastava, HM: The multiple Hurwitz zeta function and the multiple Hurwitz-Euler eta function. Taiwan. J. Math. 15(2), 501-522 (2011)

6. Srivastava, HM, Choi, J: Series Associated with Zeta and Related Functions. Kluwer Academic, Dordrecht (2001)

7. Srivastava, HM: Some generalizations and basic (or q-) extensions of the Bernoulli, Euler and Genocchi polynomials. Appl. Math. Inf. Sci. 5, 390-444 (2011)

8. Kanemitsu, S, Katsurada, M, Yoshimoto, M: On the Hurwitz-Lerch zeta function. Aequ. Math. 59, 1-19 (2000)

9. Nakamura, T: Some formulas related to Hurwitz-Lerch zeta functions. Ramanujan J. 21(3), 285-302 (2010)

10. Choi, J: Explicit formulas for Bernoulli polynomials of order $n$. Indian J. Pure Appl. Math. 27, 667-674 (1996)

11. Luo, Q-M: Some formulas for Apostol-Euler polynomials associated with Hurwitz zeta function at rational arguments. Appl. Anal. Discrete Math. 3, 336-346 (2009)

12. Liu, G-D, Srivastava, HM: Explicit formulas for the Nörlund polynomials $B_{n}^{(x)}$ and $b_{n}^{(x)}$. Comput. Math. Appl. 51(9-10), 1377-1384 (2006)

13. Garg, M, Jain, K, Srivastava, HM: Some relationships between the generalized Apostol-Bernoulli polynomials and Hurwitz-Lerch Zeta functions. Integral Transforms Spec. Funct. 17, 803-815 (2006)

14. Petojević, A: A new sum of products of Bernoulli numbers. Integral Transforms Spec. Funct. 19, 105-114 (2008)

15. Petojević, A, Srivastava, HM: Computation of Euler's sums of the products of Bernoulli numbers. Appl. Math. Lett. 22, 796-801 (2009)

16. Lewin, L: Polylogarithms and Associated Functions. North-Holland, New York (1981)

17. Zagier, D: Special values and functional equations of polylogarithms. In: Lewin, L (ed.) Structural Properties of Polylogarithms, Appendix A. Am. Math. Soc., Providence (1991)

18. Srivastava, HM, Chaudhry, MA, Qadir, A, Tassaddiq, A: Some extensions of the Fermi-Dirac and Bose-Einstein functions with applications to the family of the zeta and related functions. Russ. J. Math. Phys. 18(1), 107-121 (2011) 